\title{
Probabilities of Finding Isolated, Semi-Open, and Through Pores in Porous Polymeric Membrane Prepared by Micro-Phase Separation Method
}

\author{
Sei-ichi MANABE, Hideki IIJIMA, and Kenji KAMIDE* \\ Fundamental Research Laboratory of Fibers and Fiber-Forming Polymers, \\ Asahi Chemical Industry Co., Ltd., 11-7 Hacchonawate, \\ Takatsuki, Osaka 569, Japan
}

(Received September 16, 1987)

\begin{abstract}
The existing probabilities of isolated $\left(P_{\mathrm{i}}\right)$, semi-open $\left(P_{\mathrm{s}}\right)$, and through pores $\left(P_{\mathrm{t}}\right)$ in the micro-porous polymeric membrane were evaluated by analyzing the experimental porosity $\left(P_{\text {re }}\right)$ data. Here, the following assumptions were employed: (1) the membrane consisted of the multi-thin layers, (2) the network-like structure in one layer was constituted by numerous secondary particles which grew from the primary particles generated as polymer-rich phase from the casting solution in the occurrence of the micro-phase separation, (3) the secondary particles were packed in the hexagonal closest packed model and the vacant space originating from the polymer-lean phase was composed of imaginary vacant particles having the same diameter as the secondary particle. The probability that the secondary particles surround the vacant particles perfectly corresponds to $P_{\mathrm{i}}$, and the probability of connecting with each vacant particles continuously from the front surface to back surface equals $P_{\mathrm{t}}$. The theoretical relations between $P_{\mathrm{i}}, P_{\mathrm{s}}, P_{\mathrm{t}}$, and the structural parameters were derived. The numerical calculations were carried out for a given $P_{\mathrm{re}}$ using the equations derived. It was concluded that $P_{\mathrm{i}} \gg P_{\mathrm{s}}>P_{\mathrm{t}}$ for $P_{\mathrm{re}} \leqq 0.15$, and $P_{\mathrm{s}} \gg P_{\mathrm{i}}>P_{\mathrm{t}}$ for $0.15<P_{\mathrm{re}}<$ $0.4, P_{\mathrm{t}}>P_{\mathrm{s}}$ and $P_{\mathrm{i}}=0$ for $P_{\mathrm{re}} \geqq 0.4$.
\end{abstract}

KEY WORDS Polymeric Membrane / Micro-Phase Separation Method / Pore Characteristics / Isolated Pore / Semi-Open Pore / Through Pore / Porosity / Secondary Particle / Layer Structure /

Very recently Kamide and Manabe $^{1}$ presented a particle-growth theory for interpreting pore formation when a membrane is produced through the phase separation phenomena of polymer solution. The validity of their theory was experimentally confirmed for cellulose cuprammonium solutions and cellulose membranes regenerated from them: The pore size distribution $N(r)$ ( $r$, pore radius) of the regenerated cellulose membrane surface, indirectly estimated by the theory using the two-phase volume ratio of the phase separated solution and the average diameter of the secondary particles constitut- ing the membrane cast from the solution, agrees fairly well with the pore size distribution directly determined by the electron microscopic (EM) method proposed before. ${ }^{2}$ The phase-separation proceeded from the top to bottom surface for the case of cellulose cuprammonium solution. ${ }^{3}$ Although the overall supermolecular structure changes significantly depending on the distance $Z$ from the top surface of the membrane, it was ascertained that within a given thin layer with constant $Z$ the particular supermolecular structure of the layer remained almost uniform. ${ }^{3}$ Accordingly, $N(r)$ for each portion of

* To whom all correspondence should be addressed. 
the ultra-thin layer was constant for a given $Z$.

These experimental facts clearly lead us to the following concept that the porous polymeric membrane prepared by the micro-phase separation method should be considered as a composite, in which many hypothetical ultrathin layers are piled up and when the polymer concentration is lower than the critical concentration, the ultra-thin layer is twodimentionally composed of many small particles.

Up to now, mean pore size has widely been estimated by a water-flow-rate method $^{4}$ or a gas permeation method, ${ }^{5}$ assuming that all existing pores can be approximated with straight-through cylinders. This assumption is evidently unrealistic and unacceptable and in consequence, the physical meaning of mean pore size by a flow-rate method is unavoidably obscure. We can determine the size of the twodimentional pores and its distribution on the top and bottom surfaces and on ultra-thin layer sections of the membrane by the EM method, ${ }^{3}$ but $N(r)$ for the three-dimentional pores cannot be evaluated by this method. Of course, $N(r)$ by a bubble point method ${ }^{2,4}$ deviates significantly from the mean pore size calculated from two-dimentional $N(r)$ by the EM method, because in the former only through pores are investigated and in the

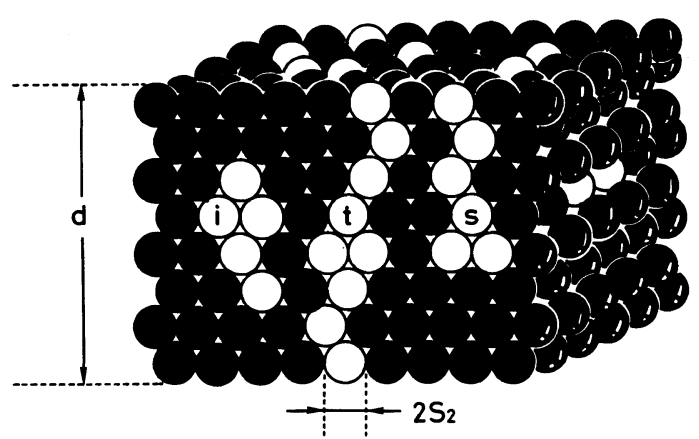

Figure 1. Schematic representation of a membrane structure composed of secondary and vacant particles. Filled sphere, secondary particle, unfilled sphere, vacant particle, diameters of these spheres are $2 S_{2}$. The symbols of $i, s$ and $t$ stand for isolated, semi-open and through pores, respecrively. latter all possible pores such as isolated, semiopen and through pores are taken into consideration (Figure 1). Then we come to a fundamental question: What is the pore in a real polymer membrane?

In this article, we present an approximate theory for evaluating the existing probabilities of isolated, semi-open and through pores $P_{\mathrm{i}}$, $P_{\mathrm{s}}$, and $P_{\mathrm{t}}$ from experimental two-dimentional porosity $P_{\text {re }}$ data by the EM method and to calculate, using the theory, numerous pore characteristics including $P_{\mathrm{i}}, P_{\mathrm{s}}$, and $P_{\mathrm{t}}$ as function of $P_{\text {re }}$.

\section{THEORETICAL BACKGROUND}

Assume that (1) the membrane is a porous membrane, (2) the membrane consists of the multi-layers, ${ }^{3}(3)$ the network-like structure in a given layer is constituted of secondary particles formed by amalgamating the primary particles generated in the initiation of the micro-phase separation, ${ }^{1}$ (4) the diameter of the secondary particles is almost constant $\left(=2 S_{2}\right)$, and (5) the secondary particles are packed in a given layer by the hexagonal closest packed model. Figure 1 shows a schema of the membrane model, in which the membrane is represented as a mixture of secondary polymer particles (filled sphere) and imaginary vacant particles (unfilled sphere), both having the same diameter of $2 S_{2}$. Here, before washing, the polymer-lean phase is assumed to be representable by vacant particles and after washing, becomes a part of a pore. The mutual interaction energies between the secondary particles, between the vacant particles and between the secondary and vacant particles are assumed to be equivalent to each other.

When the thickness of the membrane is $d$ and the radius of a particle is $S_{2}$, the total number of layers $L$ constituting a membrane is given by

$$
L=6^{1 / 2} d / 4 S_{2}
$$


The two-dimentional porosity $P_{\text {re }}$, as determined by the EM method, for a hypothetical layer in a membrane is equivalent to the summation of existing probabilities of the through, semi-open, and isolated pores, $P_{\mathrm{t}}, P_{\mathrm{s}}$, and $P_{\mathrm{i}}$, respectively. That is, eq 2 holds

$$
P_{\mathrm{re}}=P_{\mathrm{t}}+P_{\mathrm{s}}+P_{\mathrm{i}}
$$

Equation 2 indicates that if two of these three probabilities and $P_{\text {re }}$ are known in advance, the remaining probability can be readily evaluated.

\section{Theoretical Relation between $P_{\mathrm{i}}$ and $P_{\mathrm{re}}$}

In order to derive the above relation an introduction of "the shell concept" is tremendously helpful. When we pay attention to an arbitrarily chosen particle in a membrane, the total number of the nearest neighbour particles packed around the particle in question is twelve (Figure 2a). These twelve particles make a kind of spherical shell surrounding the given particle and here after we define this shell as the first shell. The second, third, ...etc. shells can also be defined in a similar manner (Figure 2b). The radius of the first

shell $R_{1}$ is approximately $2 S_{2}$ as shown as the broken curve in Figure 2b, and in general, the radius of the $i$-th shell $R_{i}$ is approximately related to $S_{2}$ through the relation,

$$
R_{i} \simeq 2 S_{2}(2 / 3)^{1 / 2}(i-1)+2 S_{2}
$$

The total number of particles constructing the $i$-th shell, $M_{(i)}$, can be approximated as the ratio of the surface area of the shell to the cross sectional area of the secondary particle:

$$
M_{(i)}=4 \pi R_{i}{ }^{2} / \pi S_{2}{ }^{2} .
$$

Combination of eq 3 with eq 4 gives

$$
M_{(i)}=16\left\{(2 / 3)(i-1)^{2}+2(2 / 3)^{1 / 2}(i-1)+1\right\}
$$

Among $M_{(i+1)}$ particles belonging to the $i+$ 1-th shell, $m_{1(i)}$ particles are located as the nearest neighbouring particles to a given particle existing in the $i$-th shell. We assume, as noted, hexagonal closest packing, and then $m_{1(i)}$ is given by

$$
m_{1(i)}=3 M_{(i+1)} / M_{(i)} \text { for } \quad M_{(i)} \neq \infty
$$

At the limit of $M_{(i+1)}=\infty$ (i.e., $R_{i+1}=\infty$ ), eq 6 can be simply reduced to

(b)

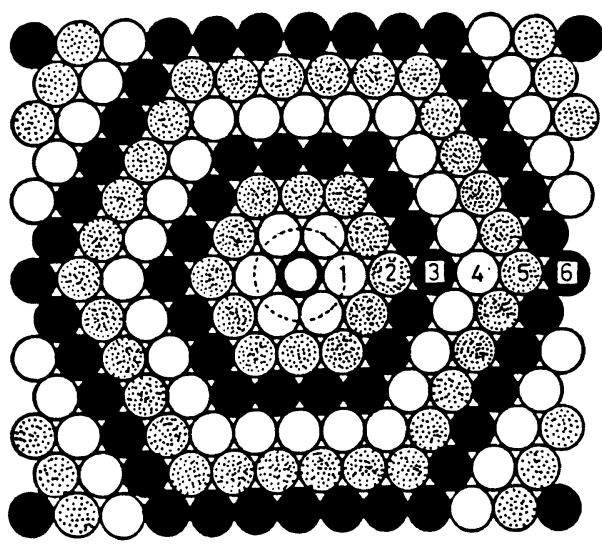

Figure 2. Schematic representation of "the shell structure." (a): A photograph of a model of the first shell. A particle located in the center of the shell cannot be seen. (b): Cross sectional view of shells. Thick full line stands for a given vacant particle in the center of the shell structure. Figures indicate shell order. Dotted line indicates a region of the first shell having a radius of $2 S_{2}$. 


$$
m_{1(i)}=3 \text { for } \quad M_{(i)}=\infty
$$

The maximum number of the particles in the $i+1$-th shell, contacting directly with $l_{(i)}$ particles, which are chosen arbitrarily from total particles in the $i$-th shell (Here, we assume $\left.l_{(i)} \ll M_{(i)}\right), m_{l(i)}$ is

$$
\begin{array}{r}
m_{l(i)}=3 l_{(i)} M_{(i+1)} / M_{(i)} \\
\text { for } l_{(i)}<M_{(i)} / 3
\end{array}
$$

Since $m_{l(i)}$ should be an integer, we round off the fraction in the right side of eq 7 to integer.

The total number of secondary and vacant particles in a unit volume of a membrane $N_{0}$ is calculated by the approximate relation,

$$
N_{0}=3 /\left\{4\left(\pi S_{2}{ }^{3}\right)\right\}
$$

An isolated pore should be surrounded completely by secondary particles. First, we consider cases in which an isolated pore is constituted with a vacant particle, two vacant particles and three vacant particles. Considering the probability that twelve possible seats around a given particle are occupied by vacant particles or secondary particles, we derive the equations of the total number of isolated pores in the unit volume of the membrane for each case. Finally, a general expression to give the total number of the isolated pores having $n_{0}$ vacant particles in the unit volume of the membrane is derived.

The total number of isolated pores, formed by only one vacant particle, in a unit volume of a membrane, $N_{1}$, is given as the products of $N_{0}$ and the probability that a particle chosen arbitrarily in the membrane is vacant $\left(=P_{\text {re }}\right)$ and the probability that all the twelve neighboring particles against that vacant particle are the secondary particles $\left(=\left(1-P_{\mathrm{re}}\right)^{12}\right)$, that is

$$
N_{1}=N_{0} P_{\text {re }} q^{12}
$$

with

$$
q=1-P_{\mathrm{re}}
$$

The total number of isolated pores, which are formed by two consecutive vacant particles in a unit volume of a membrane, $N_{2}$, is given by the product of (1) $N_{0}$ and (2) the combinatory, ${ }_{12} C_{1}$, to choose one particle from twelve particles in the first shell and (3) the probability that the particle chosen thus is vacant $\left(=P_{\text {re }}\right)$ and $(4)$ the probability that all remaining eleven particles in the abovementioned first shell are secondary particles $\left(=q^{11}\right)$ and (5) the probability that the second shell particles contacting directly the first shell vacant particle are all secondary particles $\left(=q^{m_{1(1)}}\right)$. That is,

$$
N_{2}=N_{0} \cdot{ }_{12} C_{1} \cdot P_{\mathrm{re}}^{2} q^{11} q^{m_{1(1)}}
$$

The total number of isolated pores, each made of three consecutive vacant particles in a unit volume of a membrane, $N_{3}$, is approximately given by

$$
\begin{aligned}
N_{3}= & N_{0}\left\{{ }_{12} C_{2} P_{\mathrm{re}}{ }^{3} q^{10} q^{m_{1(1)}} q^{m_{1(1)}}\right. \\
& \left.+{ }_{12} C_{1} \cdot{ }_{m_{1(1)}} C_{1} \cdot P_{\mathrm{re}}{ }^{3} q^{11} q^{m_{1(1)}-1} q^{m_{1(2)}}\right\}
\end{aligned}
$$

The detailed derivation of eq 12 is given in Appendix I.

The total number of isolated pores, each made of $n_{0}$ consecutive vacant particles in a unit volume of a membrane, $N_{n_{0}}$ for $n_{0}<13$ is derived in a similar manner as in deriving eq $9,10,11$, and 12 . The result is

$$
\begin{aligned}
N_{n_{0}}= & N_{0} \cdot q^{\left(13-n_{0}\right)}(1-q)^{n_{0}} \sum_{j(1)=0}^{12}{ }_{12} C_{j(1)} \cdot A_{j(1)} \\
& \text { for } n_{0}<13
\end{aligned}
$$

where $A_{j(1)}$ is the probability that $j_{(1)}$ vacant particles among $n_{0}$ consecutively connected particles are found in the first shell and ${ }_{12} C_{j(1)}$ stands for the combinatory of choice of $j_{(1)}$ from twelve. $A_{j(1)}$ is given by the following recurrent equation:

$$
\begin{aligned}
A_{j(1)}= & \sum_{j_{(2)}(2)=1}^{m_{1(1)}} m_{1(1)} C_{j_{(2)}(2)} A_{j_{(1)}(1), j_{(2)}(2)} q^{m_{1(1)}-j_{(2)}(2)} \\
& +q^{m_{1(1)}}
\end{aligned}
$$

$A_{j_{(1)}(1), j_{(2)(2)}}$ is very complicated and hereafter 
we employ $A_{j(1), j(2)}$.

$$
\begin{gathered}
A_{j(1),(2)}=0 \quad \text { for } j_{(2)}=0 \\
A_{j(1), j(2)}=\sum_{j(3)=1}^{m_{1(2)}} m_{1(2)} C_{j(3)} \cdot A_{j(1), j(2), j(3)} q^{m_{1(2)}-j(3)} \\
+q^{m_{1(2)}} \quad \text { for } \quad j_{(2)} \neq 0
\end{gathered}
$$

Generalized form of $A_{j_{(1)(1), j_{(2)(2)}}}$ in eq 14 should be $A_{j_{(1)}(1), \cdots, j_{(i-1)}(i-1), j_{(i)}(i)}, \cdots$, and its much simpler expression is $A_{j(1)}, \cdots, j(i-1), j(i)$.

$A_{j(1)}, \cdots, j(i-1), j(i), \ldots$ is the probability that $j_{(1)}, \cdots, j_{(i-1)}, j_{(i)}, \cdots$ vacant particles, all belonging to a pore consisting of $n_{0}$ consecutively connected particles, are found in the first, $\cdots$, the $i-1-$ th, the $i$-th $\cdots$ shells, respectively, as given by

$$
\begin{aligned}
& A_{j(1)}, \cdots, j(i-1), j(i), j(i+1), \cdots=0 \\
& j_{(i+1)}=j_{(i+2)}=\cdots=j_{(m)}=0 \text { for } j_{(i)}=0
\end{aligned}
$$

In general,

$$
\begin{aligned}
& A_{j(1), j(2), \cdots, j(m-1)} \\
& =\sum_{j(m)=1}^{m_{l(m-1)}} m_{l(m-1)} C_{j(m)} \cdot A_{j(1), j(2), \cdots, j(m)} \\
& \quad \times q^{m_{l(m-1)}-j(m)}+q^{m_{l(m-1)}} \text { for } j_{(i)} \neq 0
\end{aligned}
$$

The maximum order $\tilde{m}$ of the shells, in which at least one vacant particle of any isolated pore having $n_{0}$ sequential vacant particles is found, can be determined by eq 18

$$
\sum_{i=1}^{\tilde{m}} j_{(i)}=n_{0}-1
$$

Obviously, $\tilde{m}$ is less than $n_{0}$ :

$$
\tilde{m} \leqq n_{0}-1
$$

The total number of vacant particles contributing to formation of the isolated pores, made of $n_{0}$ consecutive vacant particles is $n_{0} N_{n_{0}}$ and the summation of $n_{0} N_{n_{0}}$ over all possible $n_{0}$ in a unit volume of a membrane, coincides with $P_{\mathrm{i}}$ :

$$
P_{\mathrm{i}}=\sum_{n_{0}=1}^{N_{0} P_{\text {re }}} n_{0} N_{n_{0}} / N_{0}
$$

Using eq 13 to 18 and 20, we can calculate $P_{\mathrm{i}}$ as a function of $P_{\text {re }}$ (accordingly, $q$ ).

\section{Theoretical Relation between $P_{\mathrm{t}}$ and $P_{\mathrm{re}}$}

In order to derive a theoretical relation of $P_{\mathrm{t}}$ as a function of $P_{\text {re }}$, the multi-thin layer model (Figure 1) is more convenient than the shell concept employed in the preceding section since the through pore must penetrate, even if (a)

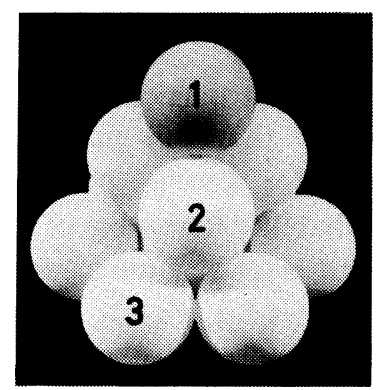

(b)

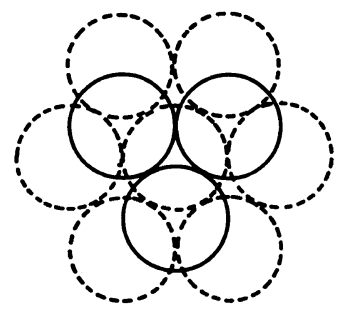

(c)

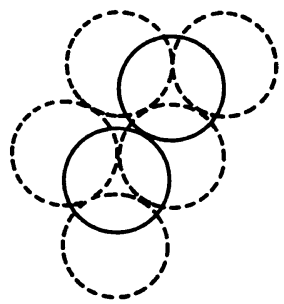

(d)

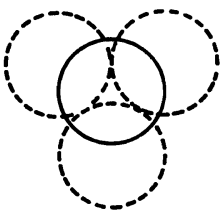

Figure 3. Some configurational patterns of the arrangement of vacant and secondary particles when a site of the first layer is occupied by a vacant particle: full line, vacant or secondary particle on the second layer; broken line, vacant or secondary particle on the third layer. (a): A photograph of a part of the layer structure model. Figures indicate layer order. Only four 3rd layer particles are seen. (b): Configuration of seven particles on the third layer neighbouring with three particles on the second layer. (c): Configuration of five particles on the third layer neighbouring with two particles on the second layer. (d): Configuration of three particles on the third layer neighbouring with one particle on the second layer. 
by a roundabout route, from the front surface to the back surface of a membrane. When a particle in the first layer is a vacant particle, three vacant or secondary particles in the 2 nd layer contact directly to the above-mentioned vacant particle and the three particles in the 2nd layer, seven vacant or secondary particles in the 3rd layer contact directly. This situation is represented by the photograph in Figure 3a.

When all three particles in the 2nd layer are vacant particles, seven 3rd layer particles contact directly these vacant particles (Figure $3 b$ ) and when two of these three 2 nd layer particles are vacant particles, five 3rd layer particles contact directly these vacant particles (Figure $3 c$ ) and when only one 2 nd layer particle is vacant particle three 3 rd layer particles contact this 2nd layer particle (Figure 3d).

Suppose an arbitrarily chosen pore at the $k$ th layer, which consists of consecutive $l_{[k]}$ vacant particles and these particles can directly contact at most $m_{l_{[k]}[k+1]}$ particles in the $k+1$ th layer. Hereafter we employ a simpler expression $m_{l[k+1]}$ instead of $m_{l_{[k]}[k+1]}$. After some tedious calculations, we obtain an approximate relation between $m_{l[k]}$ and $l_{[k]}$ :

$$
m_{l[k+1]} \simeq\left\{1+2 l_{[k]}+\left(12 l_{[k]}-3\right)^{1 / 2}\right\} / 2
$$

Here, $m_{l[k]}$ is an integer and then the value given by the left hand side of eq 21 should round off the fraction.

Consider a case when a membrane consists of two thin layers and all the pores on the first layer are constituted with only one vacant particle. Then, the existing probability of the through pore, penetrating through two neighbouring layers $P_{\mathrm{t}[2]}$ is given by the summation of these three probabilities: the probability that one of three 2 nd layer particles, contacting directly the vacant particle on the first layer is vacant $\left(=P_{\mathrm{re}}{ }_{3} C_{1} P_{\mathrm{re}} q^{2}\right)$ and the probability that two of those three 2nd layer particles mentioned above are vacant $\left(=P_{\mathrm{re}}{ }_{3} C_{2} P_{\mathrm{re}}{ }^{2} q\right)$ and the probability that all those three particles in the second layer are vacant $\left(=P_{\mathrm{re}} \cdot{ }_{3} C_{3} P_{\mathrm{re}}{ }^{3}\right)$. Then, $P_{\mathrm{t}[2]}$ is given by

$$
\begin{aligned}
P_{\mathrm{t}[2]}= & P_{\mathrm{re}} \cdot{ }_{3} C_{1} P_{\mathrm{re}} q^{2}+P_{\mathrm{re}} \cdot{ }_{3} C_{2} P_{\mathrm{re}}{ }^{2} q \\
& +P_{\mathrm{re}}{ }_{3} C_{3} P_{\mathrm{re}}{ }^{3} \\
= & P_{\mathrm{re}} \sum_{i=1}^{3}{ }_{3} C_{\mathrm{i}} P_{\mathrm{re}}{ }^{i} q^{3-i}=P_{\mathrm{re}}\left(1-q^{3}\right)
\end{aligned}
$$

By generalizing the above procedure established for a membrane with two thin layers with the case when a membrane consists of $k$ thin layers, we obtain the existing probability of the through pore $P_{\mathrm{t}[k]}$ :

$$
\begin{aligned}
P_{\mathrm{t}[k]}= & P_{\mathrm{re}} \sum_{l[2]=1}^{m_{l[2]}} m_{l[2]} C_{l[2]} P_{\mathrm{re}}^{l[2]} q^{m_{l[2]}-l[2]} \\
& \times \sum_{l[3]=1}^{m_{l[3]}}\left\{{ }_{m_{l[3]}} C_{l[3]]} P_{\mathrm{re}}^{l[3]} q^{m_{l[3]}-l[3]} \sum \cdots\right. \\
& \times \sum_{l[k-1]=1}^{m_{l l k-1]}}\left\{m_{l[k-1]} C_{l[k-1]} P_{\mathrm{re}}^{l[k-1]}\right. \\
& \times q^{m_{l[k-1]}-l[k-1]} \\
& \left.\left.\times \sum_{l[k]=1}^{m_{l[k]}}\left\{{ }^{l[k]} C_{l[k]} P_{\mathrm{re}}{ }^{l[k]} q^{m_{l[k]}-l[k]}\right\}\right\} \cdots\right\}
\end{aligned}
$$

When $k$ equals $L$, then $P_{\mathrm{t}[L]}$ corresponds to $P_{\mathrm{t}}$.

Consider the consecutive $x$ vacant particles in the first layer, constituting a part of a through pore. The existing probability of the through pore, which has $x$ vacant particles on the first layer, $P_{t}(x)$ can be approximated by

$$
P_{\mathrm{t}}(x)=\left(\pi S_{2}^{2}\right) \cdot x \cdot M(x)\left[1-\left\{1-\left(P_{\mathrm{t}[L]} / P_{\mathrm{re}}\right)\right\}^{x}\right]
$$

When we consider one vacant particle in the first layer, the probability that this particle does not become a part of a through pore is $\left\{1-\left(P_{\mathrm{t}[L]} / P_{\mathrm{re}}\right)\right\}$. The probability that none of $x$ vacant particles in the first layer become a part of through pores is $\left\{1-\left(P_{\mathrm{t}[L]} / P_{\mathrm{re}}\right)\right\}^{x}$. The probability that any of $x$ consecutive vacant particles in the first layer become a part of a through pore is $\left[1-\left\{1-\left(P_{\mathrm{t}[L]} / P_{\mathrm{re}}\right)\right\}^{x}\right]$. When we define $M(x)$ as the frequency distribution function of $x$ in the first layer, $M(x)$ can be easily transformed into the pore radius distribution function $N(r)$ in the first layer, eval- 
uated independently, through the relations:

$$
\begin{aligned}
& M(x) \mathrm{d} x=N(r) \mathrm{d} r \\
& r=x^{1 / 2} S_{2}
\end{aligned}
$$

The mean value of the existing probability of a straight-through pore, whose pore end consists of $x$ vacant particles, $P_{\mathrm{t}}(x)$, averaged over all possible $x$ as designated by $P_{t}$, is calculated by

$$
P_{t}=\pi S_{2}{ }^{2} \int x M(x)\left[1-\left\{1-\left(P_{t[\mathrm{~L}]} / P_{\mathrm{re}}\right)\right\}^{\mathrm{x}}\right] \mathrm{d} x
$$

\section{NUMERICAL CALCULATIONS}

Calculation of $P_{\mathrm{t}[k]}$ from eq 23 is very timeconsuming for large $k$. We thus derived an approximation equation of eq 23 , from which $P_{\mathrm{t}[k]}$ can be simply calculated. Since $P_{\text {re }}$ plus $q$ equals unity (see, eq 10), then $\left(P_{\mathrm{re}}+q\right)^{m_{l[k]}}(=1)$ can be expanded according to the binominal theorem. The result is:

$$
\sum_{m_{l[k]}} C_{l[k]} P_{\mathrm{re}}{ }^{k[l]} q^{m_{l[k]}-l[k]}=1-q^{m_{l[k]}}
$$

Equation 27 is a generalized equation of eq $22 b$.

Note that $m_{l[k]}$ is a function of $l_{[k-1]}$ as given by eq 21 . By substituting eq 27 into eq 23, we obtain

$$
\begin{aligned}
& P_{\mathrm{t}[k]} / P_{\mathrm{t}[k-1]}=1-\left\{\sum_{l[2]=1}^{m_{l[2]}} m_{l[2]} C_{l[2]} P_{\mathrm{re}}^{l[2]} q^{m_{l[2]}-l[2]}\right. \\
& \times \sum_{l[3]=1}^{m_{l[3]}} \cdots \sum_{l[k-1]=1}^{m_{l \mid k-1]}} m_{l[k-1]} C_{l[k-1]} \\
& \left.\times P_{\mathrm{re}}^{l[k-1]} q^{m_{l[k-1]}+m_{l[k]}-l[k-1]}\right\} / \\
& \times\left\{\sum_{l[2]=1}^{m_{l[2]}} m_{l[2]} C_{l[2]} P_{\mathrm{re}}^{l[2]} q^{m_{l[2]}-l[2]}\right. \\
& \times \sum_{l[3]=1}^{m_{l[3]}} \cdots \sum_{l[k-1]=1}^{m_{l[k-1]}} m_{l[k-1]} C_{l[k-1]} \\
& \left.\times P_{\mathrm{re}}[l k-1] q^{m_{l[k-1]}-l[k-1]}\right\}
\end{aligned}
$$

For large $k$, eq 28 reduces to

$$
P_{\mathrm{t}[k]} / P_{\mathrm{t}[k-1]} \simeq 1-(k-1)^{1 / 2} q^{k+1}
$$

The validity of eq 29 was confirmed by preliminary numerical calculations. In this article, for calculation of $P_{\mathrm{t}[k]}$ as a function of $P_{\mathrm{re}}$, eq 23 (i.e., accordingly, eq 28) was employed for $k \leqq 7$, and eq 29 for $k>7$.

\section{EXPERIMENTAL}

\section{Sample Preparation}

Cuprammonium cellulose solutions with a cellulose concentration of $5 \mathrm{wt} \%$ was cast on a flat glass plate. The cast solution on the glass plate was immersed in a coagulation solution containing acetone, ammonia and water in weight ratio of $40.0: 0.56: 59.44$ at $25^{\circ} \mathrm{C}$. After coagulation, the membrane was peeled off from the glass plate and treated with $2 \mathrm{wt} \%$ of sulfuric acid aqueous solution at $20^{\circ} \mathrm{C}$, and then washed with water. The wet membrane was immersed in acetone and then dried at $25^{\circ} \mathrm{C}$ under fixed length. The membrane thus prepared was embedded in blocked acrylic resin (mixture of $n$-butyl acrylate and methyl methacrylate) and sliced using Ultrotome ${ }^{\circledR}$ type 8800 (manufactured by LKB., Sweden) in parallel to the membrane surface to give thin sections of $1 \mu \mathrm{m}$ thickness. Only the section obtained from the top surface was employed. The acrylic resin for embedding dissolved out of the section using chloroform.

\section{Measurements}

Electron microscopic (EM) photographs on the sections were taken using a scanning electron microscope (JSM-U3 type SEM, manufactured by JEOL Co., Ltd., Japan) and $S_{2}$ and $d$ were determined directly from EM photographs. The pore radius distribution $N(r)$ was also evaluated from EM photographs using a stereological method. ${ }^{5}$ From $N(r),\left(\bar{r}_{3} \cdot \bar{r}_{4}\right)^{1 / 2}$ ( $\bar{r}_{3}$ and $\bar{r}_{4}$ are the 3 rd and 4 th mean pore radii) and $P_{\text {re }}$ were calculated. $\left(\bar{r}_{3} \cdot r_{4}\right)^{1 / 2}$ was also determined by the water-flow-rate method and denoted by $\bar{r}_{\mathrm{f}}$ to distinguish it from that by the 
EM method.

\section{RESULTS AND DISCUSSION}

Figure 4 shows the dependence of $P_{\mathrm{t}[k]}$ on the layer order $k$ constituting a membrane with a given $P_{\mathrm{re}}$. In the range $P_{\mathrm{re}}>0.9, P_{\mathrm{t}[k]}$ remains practically constant over the entire range of $k$.

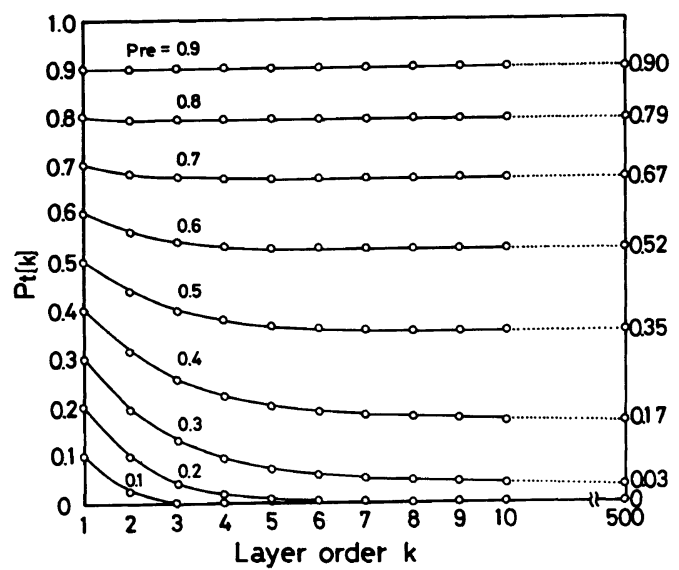

Figure 4. Layer order $k$ dependence of the existing probability of the through pore, $P_{\mathrm{t}[k]}$ with various values of $P_{\mathrm{re}}$
In the range $P_{\mathrm{re}}<0.8, P_{\mathrm{t}[k]}$ decreases first, approaching a limiting value $\left(P_{\mathrm{t}[\infty]}\right)$ with increasing $k$. In the fourth column of Table I are $P_{\mathrm{t}[\infty]}$ values estimated from Figure 4. As expected, $P_{\mathrm{t}[\infty]}$ is larger for membranes with larger $P_{\mathrm{re}}$. The $k$ value giving a level-off value of $P_{\mathrm{t}[k]}$, designated here as $k_{\mathrm{m}}$, can be roughly estimated from Figure 4 and more accurately calculated using. eq 28 . Here, we regard for convenience $P_{\mathrm{t}[k]}$ as $P_{\mathrm{t}[\infty]}$ if the condition $\quad P_{\mathrm{t}[k]} \geqq P_{\mathrm{t}[\mathrm{k}-1]} \cdot 0.9999$ holds. This condition can be rewritten in the form

$$
(k-1)^{1 / 2} q^{k+1} \leqq 0.0001
$$

The $k_{\mathrm{m}}$ values calculated thus are summarized in the second column of Table I.

With an increase in $P_{\text {re }}, k_{\mathrm{m}}$ decreases rapidly and monotonously.

In the fifth and sixth column of Table $I P_{\mathrm{s}}$ and $P_{\mathrm{i}}$ for given $P_{\text {re }}$ are listed. Here, $P_{\mathrm{i}}$ was calculated using eq 13 to 18 and 20 and $P_{\mathrm{s}}$ from $1-P_{\mathrm{t}[\infty]}-P_{\mathrm{i}}$. Hereafter, $P_{\mathrm{t}[\infty]}$ is simply denoted as $P_{\mathrm{t}}$ when there is no confusion.

For a regenerated cellulose membrane, $S_{2}$ and $d$ were found to be $0.2 \mu \mathrm{m}$ and $200 \mu \mathrm{m}$,

Table I. Pore shape characteristics determined theoretically as a function of $P_{\text {re }}$

\begin{tabular}{rrrllllll}
\hline$P_{\mathrm{re}}$ & $k_{\mathrm{m}}$ & $l_{\left[k_{\mathrm{m}}\right]}$ & $P_{\mathrm{t}[\infty]}$ & $P_{\mathrm{s}}$ & $P_{\mathrm{i}}$ & $P_{\mathrm{t}} / P_{\mathrm{re}}$ & $y$ & $\varepsilon$ \\
\hline 0.1 & 109 & 104.6 & 0.0000 & 0.0317 & 0.0683 & 0.0000 & 1.04 & 1.009 \\
0.2 & 49 & 53.4 & 0.0001 & 0.1840 & 0.0159 & 0.0005 & 1.09 & 1.012 \\
0.3 & 30 & 35.6 & 0.0321 & 0.2665 & 0.0014 & 0.107 & 1.13 & 1.014 \\
0.4 & 20 & 26.4 & 0.168 & 0.232 & 0.000 & 0.42 & 1.19 & 1.017 \\
0.5 & 15 & 20.6 & 0.352 & 0.148 & 0.000 & 0.704 & 1.24 & 1.019 \\
0.6 & 11 & 16.5 & 0.524 & 0.076 & 0.000 & 0.873 & 1.32 & 1.024 \\
0.7 & 8 & 12.4 & 0.669 & 0.031 & 0.000 & 0.956 & 1.43 & 1.029 \\
0.8 & 6 & 10.9 & 0.791 & 0.009 & 0.000 & 0.989 & 1.61 & 1.039 \\
0.9 & 4 & 8.5 & 0.899 & 0.001 & 0.000 & 0.999 & 2.04 & 1.060 \\
\hline
\end{tabular}

Table II. Pore characteristics of porous regenerated cellulose membrane

\begin{tabular}{ccccccccc}
\hline $\begin{array}{c}\text { Radius of the } \\
\text { secondary } \\
\text { particles, } S_{2} / \mu \mathrm{m}\end{array}$ & $\begin{array}{c}\text { Thickness, number of } \\
d / \mu \mathrm{m}\end{array}$ & $\begin{array}{c}\text { layers, } L \\
(\mathrm{eq} 1)\end{array}$ & $\begin{array}{c}2\left(\bar{r} \cdot \bar{r}_{4}\right)^{1 / 2} / \mathrm{nm} \\
\text { by EM method }\end{array}$ & $\begin{array}{c}2 \bar{r}_{\mathrm{f}} / \mathrm{nm} \text { by FR } \\
\text { method }^{\mathrm{a}}\end{array}$ & $\begin{array}{c}2 \bar{r}_{\mathrm{fe}} / \mathrm{nm} \\
\text { by eq } 34\end{array}$ & $\begin{array}{c}\text { Porosity, } \\
P_{\mathrm{re}} / \% \\
\text { by EM } \\
\text { method }\end{array}$ & $\begin{array}{c}\text { Probability } \\
\text { of through } \\
\text { pore, } P_{\mathrm{t}} / \%\end{array}$ & $\begin{array}{c}\text { Tortous } \\
\text { coefficient, } \\
\varepsilon(\mathrm{eq} \mathrm{33)})\end{array}$ \\
\hline 0.2 & 200 & 612 & 250 & 204 & 257 & 46.9 & 29.4 & 1.018 \\
\hline
\end{tabular}

a Water-flow-rate method. 
respectively as shown in Table II. From these values, the total number of thin layers constituting a membrane, $L$, was estimated using eq 1 as 612 (Table II). This membrane has a porosity of $46.9 \%$ by the EM method. Therefore, inspection of Figure 4 and Table I leads to the conclusion that for this cellulose membrane $L$ is almost 35 times larger than $k_{\mathrm{m}}$ (=18) and $P_{\mathrm{t}}$ was calculated to be $29.4 \%$ from the relations between $P_{\mathrm{re}}$ and $P_{\mathrm{t}[\infty]}\left(=P_{\mathrm{t}}\right)$.

As shown in Figure 5, there should be many branching pores in the $k_{\mathrm{m}}$-th layer, connected to a given vacant particle on the first layer. Considering the total number of vacant particles constituting one of the above-mentioned $k_{\mathrm{m}}$-th layer branching pores, $l_{i\left[\mathrm{k}_{\mathrm{m}}\right]}$, we define $l_{\left[k_{\mathrm{m}}\right]}$ as the summation of $l_{i\left[k_{\mathrm{m}}\right]}$ over the $k_{\mathrm{m}}$-th layer by eq 31 .

$$
l_{\left[k_{\mathrm{m}}\right]}=\sum_{i} l_{i\left[k_{\mathrm{m}}\right]}
$$

$l_{\left[k_{\mathrm{m}}\right]}$ is the total number of vacant particles in the $k_{\mathrm{m}}$-th layer, connected to a given vacant particle on the first layer.

Not all the $l_{\left[k_{\mathrm{m}}\right]}$ vacant particles have to be in contact with each other in the $k_{\mathrm{m}}$-th layer, but when the $l_{\left[k_{\mathrm{m}}\right]}$ vacant particles are assumed to be consecutive in the $k_{\mathrm{m}}$-th layer, $\left.m_{l_{\left[k_{\mathrm{m}}\right]}\left[k_{\mathrm{m}}\right.}+1\right]$ (hereafter we employ $m_{l_{\left[k_{m}+1\right]}}$, instead) may be approximately equal to the summation of the maximum number of the particles in the $k_{\mathrm{m}}+$ 1-th layer, contacting directly consecutive $l_{i\left[k_{\mathrm{m}}\right]}$ particles in the $k_{\mathrm{m}}$-th layer over the

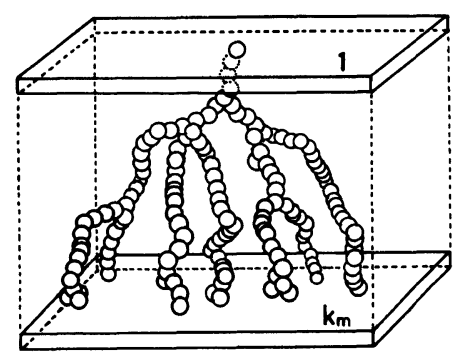

Figure 5. Schematic representation of a through pore. A through pore has one vacant particle on the first layer and many branching pores, consisting of $l_{i\left[k_{\mathrm{m}}\right]}$ consecutive vacant particles in the $k_{\mathrm{m}}$-th layer; unfilled sphere, vacant particle.

$$
\begin{gathered}
k_{\mathrm{m}}+1 \text {-th layer, } \sum_{i} m_{l_{i\left[l_{\mathrm{m}}\right]}\left[k_{\mathrm{m}}+1\right]} \text { as eq } 32 . \\
m_{l\left[k_{\mathrm{m}}+1\right]}=\sum_{i} m_{l_{i\left[k_{\mathrm{m}}\right]}\left[k_{\mathrm{m}}+1\right]}
\end{gathered}
$$

Accordingly, $m_{l\left[k_{m}+1\right]}$ should satisfy the condition given by eq 33 :

$$
q^{m_{l\left[k_{\mathrm{m}}+1\right]}} \leqq 0.0001
$$

Equation 33 was derived considering that at least one of the vacant particles on the $k_{\mathrm{m}}$-th layer connected to a given vacant particle on the first layer connects directly with vacant particle(s) on the $k_{\mathrm{m}}+1$-th layer without fail if all these vacant particles build up a through pore.

Combinating of eq 33 with eq 21 on the condition of eq 32 enables us to calculate $l_{\left[k_{\mathrm{m}}\right]}$ as a function of $P_{\mathrm{re}}$. The results are shown in the third column of Table I. For example, $l_{\left[k_{m}\right]}$ and $k_{\mathrm{m}}$ values of a membrane with $P_{\mathrm{re}}=0.5$ are estimated from Table I to be 20.6 and 15, respectively. This means that one vacant particle on the first layer is indirectly connected to 20.6 vacant particles on the 15 th layer by forming branches.

Now consider the case in which a vacant particle located on the $k$-th layer arbitrarily chosen connects directly, on average, to $y$ vacant particles on the $k+1$-th layer. If $y$ can be regarded as constant, independent of $k, y$

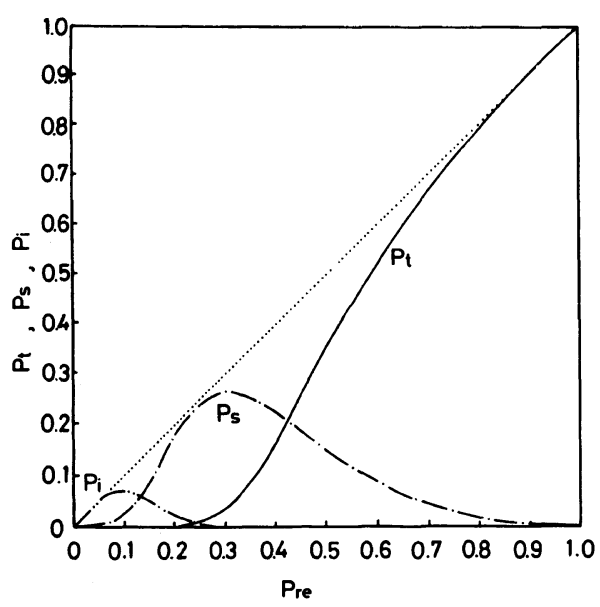

Figure 6. $P_{\mathrm{re}}$ dependence of $P_{\mathrm{t}}, P_{\mathrm{s}}$, and $P_{\mathrm{i}}$ at $k$ of 500 . 
can be calculated from $k_{\mathrm{m}}$ and $l_{\left[k_{\mathrm{m}}\right]}$ by the relation

$$
y^{k_{\mathrm{m}}-1}=l_{\left[k_{\mathrm{m}}\right]}
$$

$y$ values obtained thus for various $P_{\text {re }}$ are presented in the 8th column of Table I.

Figure 6 shows $P_{\text {re }}$ dependence of $P_{\mathrm{t}}, P_{\mathrm{s}}$, and $P_{\mathrm{i}}$ of a membrane with $L=500$. For $P_{\mathrm{re}}>0.4$, the through pores are most abundantly observed and for $0.15<P_{\mathrm{re}}<0.4$, the semi-open pores are dominant and for $P_{\mathrm{re}}<0.15$, most of the pores are the isolated pores.

The water flux $J$ is related only with the through pore. Then the mean pore radius $\bar{r}_{\mathrm{f}}$ evaluated from $J$ and porosity should be improved to $\bar{r}_{\text {fe }}$ given as follows

$$
\bar{r}_{\mathrm{fe}}=\bar{r}_{\mathrm{f}}\left(P_{\mathrm{re}} / P_{\mathrm{t}}\right)^{1 / 2} \varepsilon^{1 / 2}
$$

Here, the tortuous coefficient $\varepsilon$ is given by

$$
\varepsilon=\left\{1+(3 / 4)^{2} \cdot 6 l_{\left[k_{\mathrm{m}}\right]} / P_{\mathrm{re}}\left(1 / 4 k_{\mathrm{m}}\right)^{2}\right\}
$$

Equation 36 is derived in Appendix II.

The value of $S_{2}$ for a typical regenerated cellulose plane membrane was $0.2 \mu \mathrm{m}$ and $d$ was $200 \mu \mathrm{m}$. By substitution of these $S_{2}$ and $d$ values into eq $1, L$ was determined to be 612 . $P_{\text {re }}$ was 46.9 and $2 \bar{r}_{\mathrm{f}}$ was $204 \mathrm{~nm}$. $P_{\mathrm{t}}$ and $\varepsilon$ calculated using these experimental values are 29.4 and 1.018 , respectively. The calculated value of $2 \bar{r}_{\mathrm{fe}}$ given in eq 35 is $257 \mathrm{~nm}$. The mean pore size of $2\left(\bar{r}_{3} \cdot \bar{r}_{4}\right)^{1 / 2}$ from the EM photographs of this membrane was $250 \mathrm{~nm}$. The coincidence between $2 \bar{r}_{\mathrm{fe}}$ and $2\left(\bar{r}_{3} \cdot \bar{r}_{4}\right)^{1 / 2}$ is surprisingly excellent.

\section{APPENDIX I}

\section{Derivation of Equation 12}

In order to calculate the toral number of isolated pores, each made of three vacant particles in a unit volume of a membrane, $N_{3}$, we consider three cases for the arrangement of those three vacant particles: a), two of three vacant particles in the first shell are directly connected with each other; b), two of three vacant particles in the first shell are not di- rectly connected with each other; c), one vacant particle is located in the center of the shell structure and another vacant particle is in the first shell and the last vacant particle is in the second shell.

In the case a), the total number of the isolated pores in a unit volume of a membrane, $N_{3 \mathbf{a}}$, is given by the product of 1) $N_{0}$ and 2) the probability that one particle is vacant $\left(=P_{\mathrm{re}}\right)$, and 3) the combinatory, ${ }_{12} C_{1}$ to choose one from twelve particles in the first shell of the vacant particles, and 4) the combinatory ${ }_{12} C_{1} \cdot{ }_{4} C_{1} / 2$, to choose consecutively connected two first shell particles from twelve particles in the first shell, and 5) these two particles are vacant $\left(=P_{\mathrm{re}}{ }^{2}\right)$, and 6) the other ten particles in the first shell are the secondary particles $\left(=q^{10}\right)$, and 7$)$ the probability that $m_{2(1)}$ particles, which are in the second shell and neighbouring to the consecutively connected two vacant particles in the first shell, are the secondary particles $\left(=q^{m_{2(1)}}\right)$. That is,

$$
\begin{aligned}
N_{3 \mathrm{a}} & =N_{0} \cdot P_{\mathrm{re}} \cdot{ }_{12} C_{1} \cdot{ }_{4} C_{1} / 2 \cdot P_{\mathrm{re}}{ }^{2} q^{10} q^{m_{2(1)}} \\
& =N_{0} \cdot P_{\mathrm{re}}{ }_{12}^{3} C_{2} q^{10}(4 / 11) q^{m_{2(1)}}
\end{aligned}
$$

In case $b$ ), the total number of the isolated pores in a unit volume of a membrane, $N_{3 \mathrm{~b}}$, is given by the product of 1) $N_{0}$, and 2) the probability that one particle is vacant $\left(=P_{\mathrm{re}}\right)$, and 3) the combinatory to choose two particles which are not neighbouring each other from twelve particles in the first shell, $\left(={ }_{12} C_{2}-{ }_{12} C_{1} \cdot{ }_{4} C_{1} / 2=42\right)$, and 4) the probability that these two particles are vacant $\left(=P_{\mathrm{re}}{ }^{2}\right)$, and 5) the other ten particles in the first shell are the secondary particles $\left(=q^{10}\right)$, and 6) the probability that $2 m_{1(2)}$ particles, which are in the second shell and neighbouring to two not-directly-connecting vacant particles in the first shell, are the secondary particles $\left(=q^{2 m_{1(1)}}\right)$. That is,

$$
\begin{aligned}
N_{3 \mathrm{~b}}= & N_{0} \cdot P_{\mathrm{re}} \cdot P_{\mathrm{re}}^{2}\left({ }_{12} C_{2}-{ }_{12} C_{1} \cdot{ }_{4} C_{1} / 2\right) \\
& \times q^{10} q^{2 m_{1(1)}} \\
= & N_{0} \cdot P_{\mathrm{re}}{ }^{3}{ }_{12} C_{2} q^{10}(7 / 11) q^{2 m_{1(1)}}
\end{aligned}
$$


In case c), the total number of the isolated pores in a unit volume of a membrane, $N_{3 c}$, is given by the product of 1) $N_{0}$, and 2) the probability that one particle is vacant $\left(=P_{\mathrm{re}}\right)$, and 3 ) the combinatory to choose one from twelve particles in the first shell, and 4) the probability that this particle is vacant $\left(=P_{\mathrm{re}}\right)$, and 5) the other eleven particles in the first shell are the secondary particles $\left(=q^{11}\right)$, and 6) the combinatory to choose one from $m_{1(1)}$ second shell particles, which are neighbouring to the vacant particles in the first shell, ${ }_{m_{1(1)}} C_{1}$, and 7) the probability that this particle in the second shell is vacant $\left(=P_{\mathrm{re}}\right)$, and 8$)$ the other $\left(m_{1(1)}-1\right)$ particles are secondary particles, $q^{\mathrm{m}_{1(1)}-1}$, and 9) the probability that all the particles, which are neighbouring to the second shell vacant particle and located in the 3rd shell, are the secondary particles $\left(=q^{\left.m_{1(2)}\right)}\right.$. That is,

$$
\begin{aligned}
N_{3 \mathrm{c}}= & N_{0} \cdot P_{\mathrm{re}} \cdot{ }_{12} C_{1} \cdot P_{\mathrm{re}} \cdot q^{11}{ }_{m_{1(1)}} C_{1} \\
& \times P_{\mathrm{re}} \cdot q^{m_{1(1)}-1} \cdot q^{m_{1(2)}} \\
= & N_{0} \cdot P_{\mathrm{re}}{ }^{3} \cdot{ }_{12} C_{1} q^{i 1}{ }_{m_{1(1)}} C_{1} \\
& \times q^{m_{1(1)}-1} q^{m_{1(2)}}
\end{aligned}
$$

The total number of the isolated pores, each made of three vacant particles in a unit volume of a membrane, $N_{3}$, is given by the summation of $N_{3 \mathrm{a}}, N_{3 \mathrm{~b}}$, and $N_{3 \mathrm{c}}$. That is,

$$
\begin{aligned}
N_{3}= & N_{0} \cdot P_{\mathrm{re}}^{3}{ }_{12} C_{2} q^{10}(4 / 11) q^{m_{2(1)}} \\
& +N_{0} \cdot P_{\mathrm{re}}^{3}{ }_{12} C_{2} q^{10}(7 / 11) q^{2 m_{1(1)}} \\
& +N_{0} \cdot P_{\mathrm{re}}{ }^{3} \cdot{ }_{12} C_{1} q^{11}{ }_{m_{1(1)}} C_{1} \\
& \times q^{m_{1(1)}-1} q^{m_{1(2)}} \\
= & N_{0} \cdot P_{\mathrm{re}}{ }^{3}{ }_{12} C_{2} q^{10}\left\{(4 / 11) q^{m_{2(1)}}\right. \\
& \left.+(7 / 11) q^{2 m_{1(1)}}\right\} \\
& +N_{0} \cdot P_{\mathrm{re}}{ }^{3} \cdot{ }_{12} C_{1} q^{11}{ }_{m_{1(1)}} \mathrm{C}_{1} \\
& \times q^{\mathrm{m}_{1(1)}-1} q^{m_{1(2)}}
\end{aligned}
$$

As $m_{2(1)}$ is nearly equal $2 m_{1(1)}$, eq A-4 is rewritten to obtain eq 12 .

$$
\begin{aligned}
N_{3}= & N_{0}\left\{{ }_{12} C_{2} \cdot P_{\mathrm{re}}{ }^{3} q^{10} q^{2 m_{1(1)}}\right. \\
& \left.+{ }_{12} C_{1} \cdot{ }_{m_{1(1)}} C_{1} \cdot P_{\mathrm{re}} q^{3} q^{11} q^{m_{1(1)}-1} q^{m_{1(2)}}\right\}
\end{aligned}
$$

\section{APPENDIX II}

\section{Derivation of Equation 36}

Figure A-1 shows a schematic representation of the perspective view of the through pore having one vacant particle on the first layer. We draw a line from the vacant particle on the first layer to any vacant particle distributed from the 2 nd to $k_{\mathrm{m}}$-th layers parallel to the first layer. $\theta$ is an angle between this line and a perpendicular line, penetrating the vacant particles on the first layer, against a layer. The area of the circle including all vacant particles on the $k_{\mathrm{m}}$-th layer which is connected to the given vacant particle on the first layer is approximated by $l_{\left[k_{\mathrm{m}}\right]} \pi S_{2}{ }^{2} / P_{\mathrm{re}}$ and then the radius of this circle, $R_{m}$, is $S_{2}\left(l_{\left[k_{\mathrm{m}}\right]} / P_{\mathrm{re}}\right)^{1 / 2}$. Since $L_{\mathrm{m}}$ is given by $4 S_{2} k_{\mathrm{m}} /$ $6^{1 / 2}$, the maximum $\tan \theta, \tan \theta_{\mathrm{m}}$, equals $R_{\mathrm{m}} /$ $L_{\mathrm{m}}$. Equation (A-4) holds

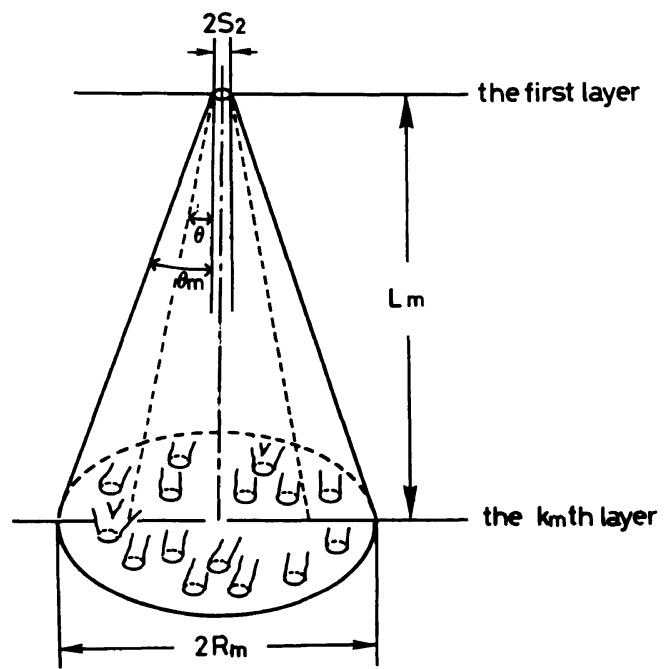

Figure A-1. Schematic representation of the perspective view of the through pore: Vacant particles on the $k_{\mathrm{m}}-$ th layer, which can connect with one vacant particle on the first layer, included in a circle having a diameter of $2 R_{\mathrm{m}}$. 


$$
\tan \theta_{\mathrm{m}}=R_{\mathrm{m}} / L_{\mathrm{m}}=\left(6 l_{\left[k_{\mathrm{m}}\right]} / P_{\mathrm{re}}\right)^{1 / 2} / 4 k_{\mathrm{m}}
$$

The mean value of $\tan \theta, \overline{\tan \theta}$ is calculated by eq (A-6)

$$
\begin{aligned}
\overline{\tan \theta}= & \int_{0}^{\tan \theta_{\mathrm{m}}}(\tan \theta)(\tan \theta)^{2} \mathrm{~d}(\tan \theta) / \\
& \times \int_{0}^{\tan \theta_{\mathrm{m}}}(\tan \theta)^{2} \mathrm{~d}(\tan \theta) \\
= & 3 \tan \theta_{\mathrm{m}} / 4
\end{aligned}
$$

The definition of the tortous coefficient $\varepsilon$ leads to the following equation

$$
\begin{aligned}
\varepsilon & =\left\{L_{\mathrm{m}}{ }^{2}+L_{\mathrm{m}}{ }^{2}(\overline{\tan \theta})^{2}\right\}^{1 / 2} / L_{\mathrm{m}} \\
& =\left\{1+(\tan \theta)^{2}\right\}^{1 / 2} \\
& =\left\{1+\left(3 \tan \theta_{\mathrm{m}} / 4\right)^{2}\right\}^{1 / 2} \\
& =\left\{1+(3 / 4)^{2} 6 l_{\left[k_{\mathrm{m}}\right]} / P_{\mathrm{re}} /\left(4 k_{\mathrm{m}}\right)^{2}\right\}^{1 / 2}
\end{aligned}
$$

\section{GLOSSARY OF SYMBOLS}

$A_{j(i)}$, probability that $j_{(i)}$ vacant particles among $n_{0}$ consecutively connected vacant particles are found in the $i$-th shell

$A_{j(1)}$, probability that $j_{(1)}$ vacant particles among $n_{0}$ consecutively connected vacant particles are found in the first shell

$A_{j(1), j(2), \cdots, j(i-1), j(i)}$, probability that $j_{(1)}, j_{(2)}$, $\cdots, j_{(i-1)}, j_{(i)}$ vacant particles, all belonging to a pore consisting of $n_{0}$ consecutively connected particles, are found in the first, second, $\cdots, i-1$-th, $i$-th shell, respectively

$d$, thickness of a membrane

$i$, order of shells

$j_{(i)}$, total number of vacant particles in the $i$-th shell

$J$, water flux

$k$, layer order

$k_{\mathrm{m}}$, minimum $k$ giving the level-off $P_{\mathrm{t}[k]}$ value $l_{(i)}$, number of particles which are chosen arbitrarily from the $i$-th shell

$l_{i\left[k_{\mathrm{m}}\right]}$, number of the consecutive vacant particles belonging to one of the branching pores at the $k_{\mathrm{m}}$-th layer, which are connected to a given vacant particle on the first layer

$l_{[k]}$, number of consecutive vacant particles in the $k$-th layer

$l_{\left[k_{\mathrm{m}}\right]}$, number of the vacant particles at the $k_{\mathrm{m}}$ th layer, which are connected to a given vacant particle on the first layer $\left(=\sum_{i} l_{i\left[k_{\mathrm{m}}\right]}\right)$

$L$, total number of layers constituting a membrane

$\tilde{m}$, the maximum order of shells

$m_{l(i)}$, the maximum number of the particles in the $i+1$-th shell in contact directly with $l_{(i)}$ particles, which are chosen arbitrarily from all particles in the $i$-th shell

$m_{l_{i\left[k_{m}\right]}\left[k_{m}+1\right]}$, the maximum number of the particles in the $k_{\mathrm{m}}+1$-th layer in contact directly with consecutive $l_{i\left[k_{\mathrm{m}}\right]}$ particles of the $i$-th pore in the $k_{\mathrm{m}}$-th layer

$m_{l[k]}$, the maximum number of the particles in the $k$-th layer, contacting directly consecutive $l_{[k-1]}$ vacant particles in the $k-1$-th layer, $m_{l_{[k-1]}[k]}$

$m_{l_{\left[k_{m}\right]}\left[k_{m}+1\right]}$, the maximum number of the particles in the $k_{\mathrm{m}}+1$-th layer, contacting directly consecutive $l_{\left[k_{\mathrm{m}}\right]}$ vacant particles in the $k_{\mathrm{m}}$-th layer

$m_{1(i)}$, total number of the partices, belonging to the $i+1$-th shell and located as the nearest neighbouring particle compared to a given particle existing in the $i$-th shell

$M(i)$, total number of the particles constituting the $i$-th shell

$M(x)$, frequency distribution function of pore which is made of $x$ consecutive vacant particles

$n_{0}$, number of the consecutively connected vacant particles, which constitute isolated pores

$N_{n_{0}}$, total number of the isolated pores, each made of $n_{0}$ consecutive vacant particles in a unit volume of a membrane

$N(r)$, pore radius distribution function

$N_{0}$, total number of secondary and vacant patricles in a unit volume of a membrane

$N_{1}$, total number of the isolated pores, which are formed by only one vacant particle, in a 
unit volume of a membrane

$N_{2}$, total number of the isolated pores, which are formed by two consecutive vacant particles in a unit volume of a membrane

$N_{3}$, total number of the isolated pores, each made of three vacant particles in a unit volume of a membrane

$N_{3 \mathrm{a}}$, total number of the isolated pores constituted by three vacant particles, two of which directly connect to each other, in a unit volume of a membrane

$N_{3 \mathrm{~b}}$, total number of the isolated pores constituted by three vacant particles, two of which are not directly connected to each other, in a unit volume of a membrane

$N_{3 \mathrm{c}}$, total number of the isolated pores constituted by three vacant particles, one is in the first shell and another is in the second shell, in a unit volume of a membrane

$P_{\mathrm{i}}$, existing probability of isolated pore

$P_{\text {re }}$, two dimentional porosity by the EM method in a unit area of a membrane surface or hypothetical thin layer

$P_{\mathrm{s}}$, existing probability of a semi-open pore

$P_{t}$, existing probability of a through pore

$P_{\mathrm{t}[k]}$, existing probability of the through pore when a membrane consists of $k$ thin layers and all the pores on the first layer are constituted by only one vacant particle

$P_{t}(x)$, existing probability of the though pore, which ends at a given pore and made of $x$ consecutive vacant particles in the first layer

$P_{\mathrm{t}[2]}$, existing probability of the through pore, penetrating through two neighbouring layers when a membrane is consisted of two thin layers and all the pores on the first layer are constituted by only one vacant paticle

$P_{\mathrm{t}[\infty]}, P_{\mathrm{t}[k]}$ at the limit of $k \rightarrow \infty$

$q$, probability that a secondary particle exists, which is equal to $1-P_{\text {re }}$

$r$, pore radius

$\bar{r}_{\mathrm{f}}$, mean pore radius determined by the waterflow-rate method

$\bar{r}_{\mathrm{fe}}$, improved mean pore radius obtained by considering $\varepsilon, P_{\text {re }}$ and $P_{\mathrm{t}}$

$\bar{r}_{3}$, the third mean pore radius

$\bar{r}_{4}$, the fourth mean pore radius

$R_{i}$, radius of the $i$-th shell

$S_{2}$, radius of secondary and vacant particles

$x$, number of consecutive vacant particles in the first layer forming a given pore

$y$, average number of vacant particles on the $k+1$-th layer, with which a vacant particle, arbitrarily chosen, on the $k$-th layer is connected directly

$\varepsilon$, tortous coefficient

\section{REFERENCES}

1. K. Kamide and S. Manabe, "Material Science of Synthetic Membranes,” D. R. Lloyd, Ed., American Chemical Society, Washington D. C., 1985, p 197.

2. S. Manabe, Y. Shigemoto, and K. Kamide, Polym. J., 17, 775 (1985).

3. S. Manabe, Y. Kamata, H. Iijima, and K. Kamide, Polym. J., 19, 391 (1987).

4. K. Kamide and S. Manabe, "Ultrafiltration Membranes and Applications," A.R. Cooper, Ed., Plenum Press, New York, N. Y., 1980, p 173.

5. S. Manabe, K. Kamide, T. Nohmi, and T. Kawai, Kobunshi Ronbunshu, 37, 405 (1980).

6. S. Manabe, M. Iwata, and M. Inoue, Japanese Patent Application, 58-89626 (1983). 